Supporting Information

\title{
Deciphering Erasing/Writing/Reading of Near-Infrared Fluorophore for Nonvolatile Optical Memory
}

Nuo-Hua Xie, Cheng Fan, Huan Ye, Kai Xiong, Chong Li* and Ming-Qiang Zhu*

Wuhan National Laboratory for Optoelectronics, School of Optical and Electronic Information, Huazhong University of Science and Technology, Wuhan, Hubei 430074, China.

*E-mail: mqzhu@hust.edu.cn and chongli@hust.edu.cn 


\section{Experiment Procedures}

\subsection{Instruments}

Reported yields are isolated yields. The NMR spectra were recorded using a 600M Bruker AscendTM $600 \mathrm{MHZ}$ in $\mathrm{CDCl}_{3}$ or $\mathrm{CD}_{2} \mathrm{Cl}_{2}$ and an internal standard of tetramethylsilane was used. MALDI TOF mass spectra were recorded with a MALDI-TOF/TOF (Bruker ultrafleXtreme). The purity of TDI-4DTE was obtained on a Waters Breeze 2 HPLC with UV detector (325 nm) using a $5 \mu \mathrm{m}$ CN column and eluting with DCM/hexane binary mixed solvent. Electrochemical experiments were performed on a CHI760D electrochemical workstation. The UV-Vis absorption and photoluminescence emission of the compounds were recorded on Shimadzu UV-VIS-NIR Spectrophotometer (UV-3600) and Edinburgh instruments (FLS 920 spectrometers), respectively. The relative fluorescence quantum yields were estimated using TDI in chloroform $\left(\Phi_{\mathrm{F}}=0.9\right)^{1}$ as standard. The absolute fluorescence quantum yields of solid films are measured by integrating sphere on Edinburgh instruments (FLS 980 spectrometers). The $302 \mathrm{~nm}$ ultraviolet irradiation was performed using an ultraviolet transmission platform (UVP, LLC, US, with 3 ultraviolet tubes, each $8 \mathrm{~W}$ $220 \mathrm{~V}$ ), the $621 \mathrm{~nm}$ visible light was obtained from a red GCI-060401 LED and the $720 \mathrm{~nm}$ light was gotten from a $50 \mathrm{mWcm}^{-2}$ LED. Photographs were taken with a digital Single Lens Reflex (NIKON D90). The fluorescence imaging was obtained by the home-built system consisting of an Olympus IX 71 inverted optical microscope, a 100x/NA1.49 oil immersion TIRF objective (UAPON 100XOTIRF, Olympus), a 561

nm solid-state laser (OBIS $561 \mathrm{LS}$, Coherent, $150 \mathrm{~mW}$ ) and a sCMOS camera (Andor iXon 897). Electronic shutters (UNIBLITZ VS14, Vincent Associates) were used to control the duration of laser irradiance and a dichroic mirror (DM: ZT561rdc-xr, Chroma) and a single band filter (EM: ET630/75m, Chroma) were used to separate the collected fluorescence from scattering laser and impurity fluorescence. 


\subsection{Synthesis of TDI-4DTE}

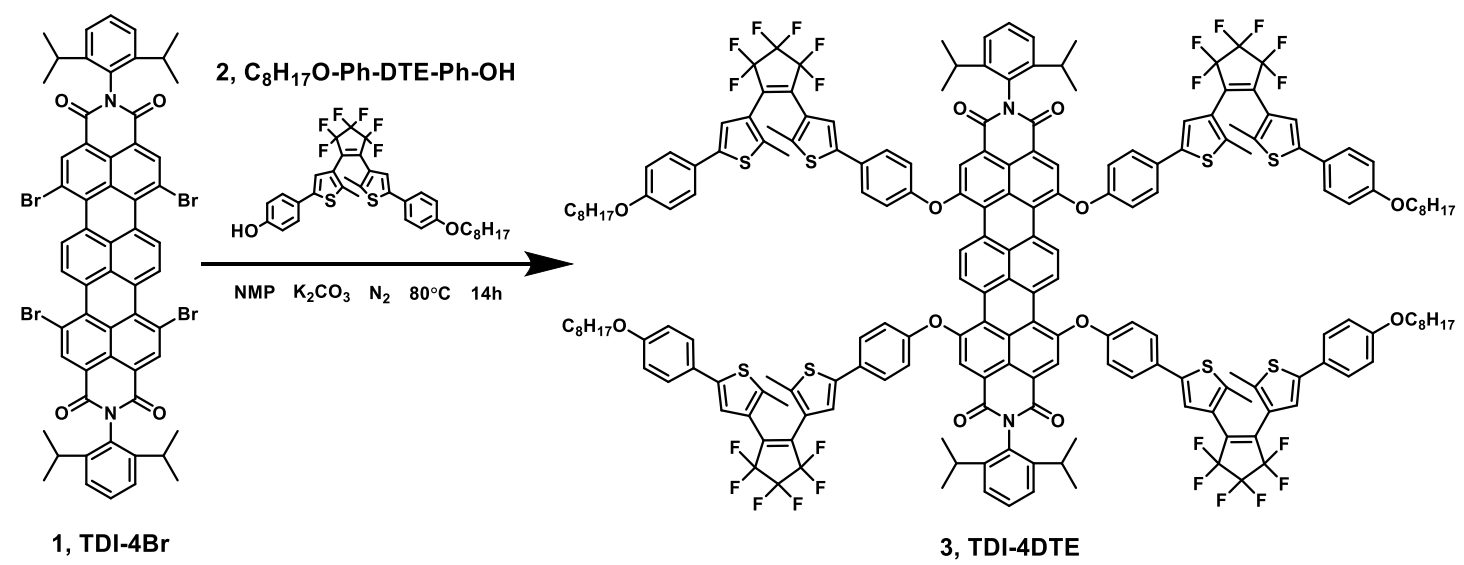

Scheme S1. Synthesis route to TDI-4DTE conjugate.

A mixture of TDI-4Br (0.33 g, $0.29 \mathrm{mmol}), \mathrm{C}_{8} \mathrm{H}_{17} \mathrm{O}-\mathrm{Ph}-\mathrm{DTE}-\mathrm{Ph}-\mathrm{OH}(1.53 \mathrm{~g}, 2.29$ $\mathrm{mmol}), \mathrm{K}_{2} \mathrm{CO}_{3}(0.20 \mathrm{~g}, 1.44 \mathrm{mmol})$ and $\mathrm{N}$-methyl-2-pyrrolidone $(50 \mathrm{~mL})$ was added and the system was stirred under nitrogen at $80{ }^{\circ} \mathrm{C}$ for $14 \mathrm{~h}$. After cooling to room temperature, water and conc. hydrochloric acid were added and the precipitate was collected by filtration, washed with water, dried under vacuum. The crude product was purified by gravity silica-gel column chromatography eluting with dichloromethane/hexane $(1: 1, \mathrm{v} / \mathrm{v})$ to yield the cyan product $(0.24 \mathrm{~g}, 24.0 \%)$.

${ }^{1} \mathrm{H}$ NMR (600 MHz, $\mathrm{CD}_{2} \mathrm{Cl}_{2}$ ), $\delta$ (ppm): 9.48 (s, 4H), 8.31 (s, 4H), 7.61 (d, J = 8.5 $\mathrm{Hz}, 8 \mathrm{H}), 7.48(\mathrm{~d}, \mathrm{~J}=7.7 \mathrm{~Hz}, 2 \mathrm{H}), 7.45$ (t, J = 7.2 Hz, 8H), 7.32 (d, J = 7.9 Hz, 4H), $7.27(\mathrm{~s}, 4 \mathrm{H}), 7.22(\mathrm{~d}, \mathrm{~J}=8.5 \mathrm{~Hz}, 8 \mathrm{H}), 7.17(\mathrm{~s}, 4 \mathrm{H}), 6.88(\mathrm{~d}, \mathrm{~J}=8.5 \mathrm{~Hz}, 8 \mathrm{H}), 3.96(\mathrm{t}, \mathrm{J}$ $=6.6 \mathrm{~Hz}, 8 \mathrm{H}), 2.70(\mathrm{dt}, \mathrm{J}=13.5,6.8 \mathrm{~Hz}, 4 \mathrm{H}), 1.95(\mathrm{~d}, \mathrm{~J}=20.0 \mathrm{~Hz}, 24 \mathrm{H}), 1.76$ (dd, J = 14.5, 7.0 Hz, 8H), $1.44(\mathrm{~d}, \mathrm{~J}=7.7 \mathrm{~Hz}, 8 \mathrm{H}), 1.32$ (dd, J = 20.8, $10.4 \mathrm{~Hz}, 32 \mathrm{H}), 1.10$ $(\mathrm{d}, \mathrm{J}=6.8 \mathrm{~Hz}, 24 \mathrm{H}), 0.88(\mathrm{t}, \mathrm{J}=6.7 \mathrm{~Hz}, 12 \mathrm{H}) .{ }^{13} \mathrm{C} \mathrm{NMR}\left(151 \mathrm{MHz}, \mathrm{CD}_{2} \mathrm{Cl}_{2}\right), \delta(\mathrm{ppm})$ : $162.88,159.10,155.30,154.19,145.58,142.35,141.40,141.03,140.22,131.18$, $130.45,130.08,129.53$, 128.91, 127.69, 126.86, 126.32, 126.05, 125.90, 125.63, 123.98, 123.25, 122.47, 122.29, 121.02, 119.72, 114.94, 77.22, 77.01, 76.80, 68.18, $31.81,29.36,29.24,29.22,29.13,26.03,24.04,22.66,14.56,14.53,14.10$. HPLC purity: 99.15\%. MS (MALDI-TOF, $\mathrm{m} / \mathrm{z}$ ): The theoretical molecular weight of $\mathrm{C}_{198} \mathrm{H}_{174} \mathrm{~F}_{24} \mathrm{~N}_{2} \mathrm{O}_{12} \mathrm{~S}_{8}$ is 3486.01 , found $3485.601\left([\mathrm{M}]^{+}\right)$ 


\section{Results and Discussion}

\subsection{Optical properties of TDI-4DTE}

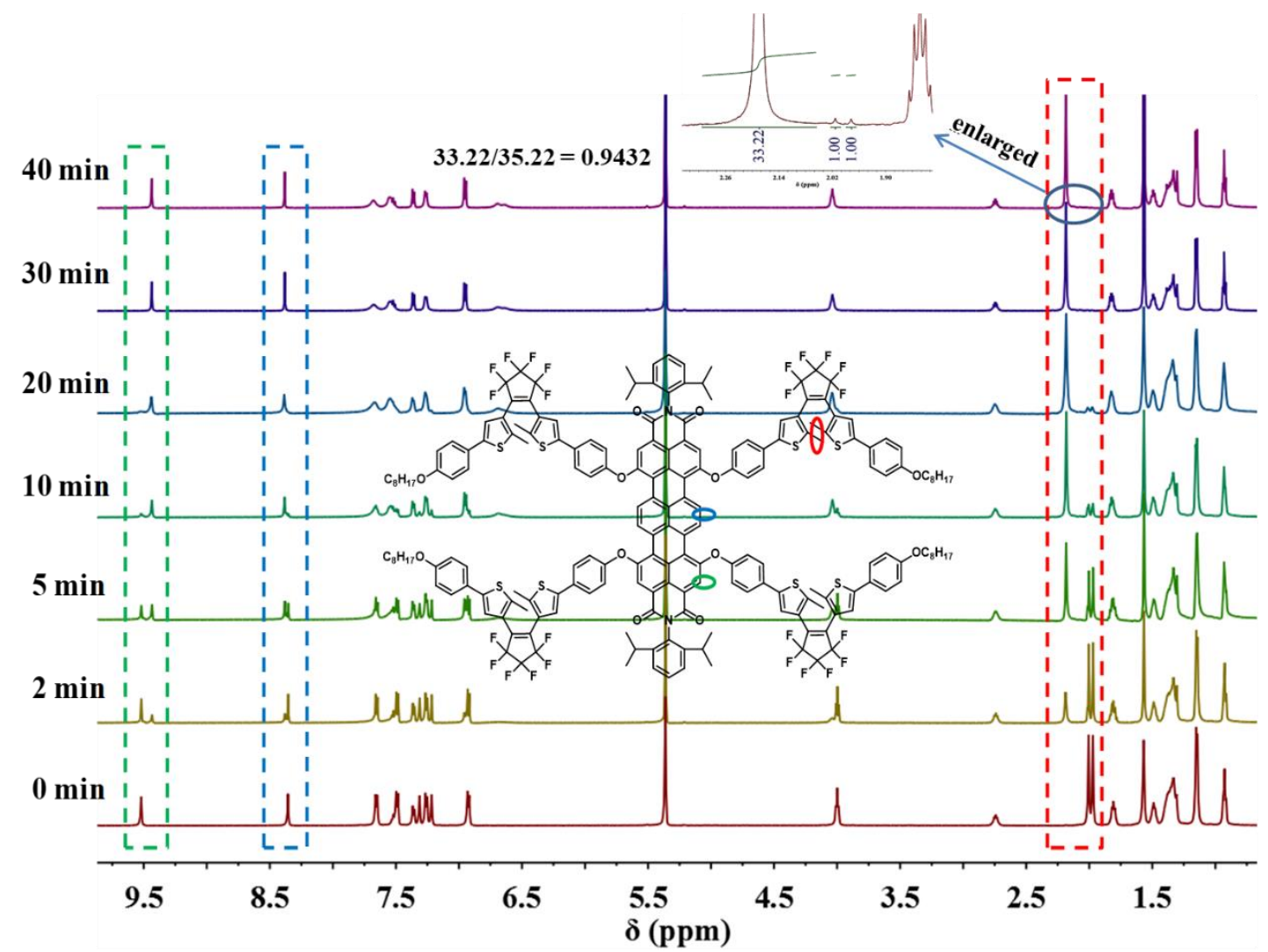

Figure S1. The conversion efficiency of TDI-4DTE at PSS is measured about $94.32 \%$ according to the integrate value change of the methyl groups on thiophene rings in ${ }^{1} \mathrm{H}$ NMR spectra before and after $302 \mathrm{~nm} \mathrm{UV}$ irradiation in $\mathrm{CD}_{2} \mathrm{Cl}_{2}$.
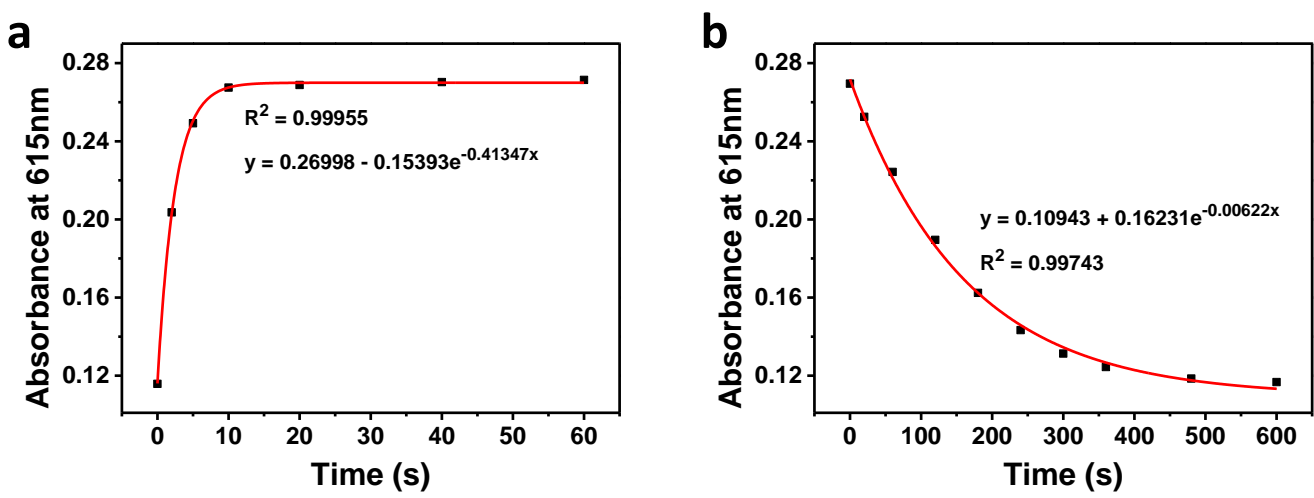

Figure S2. Time-resolved spectroscopic monitoring of (a) the forward and (b) the backward photochromic interconversions between open and closed forms of TDI-4DTE in THF. 


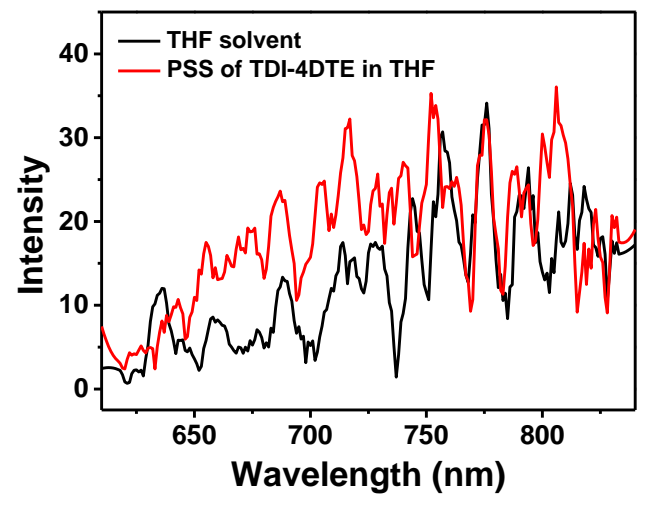

Figure S3. Fluorescence spectra of TDI-4DTE at photostationary state (PSS) in THF upon $302 \mathrm{~nm}$ UV light irradiation.
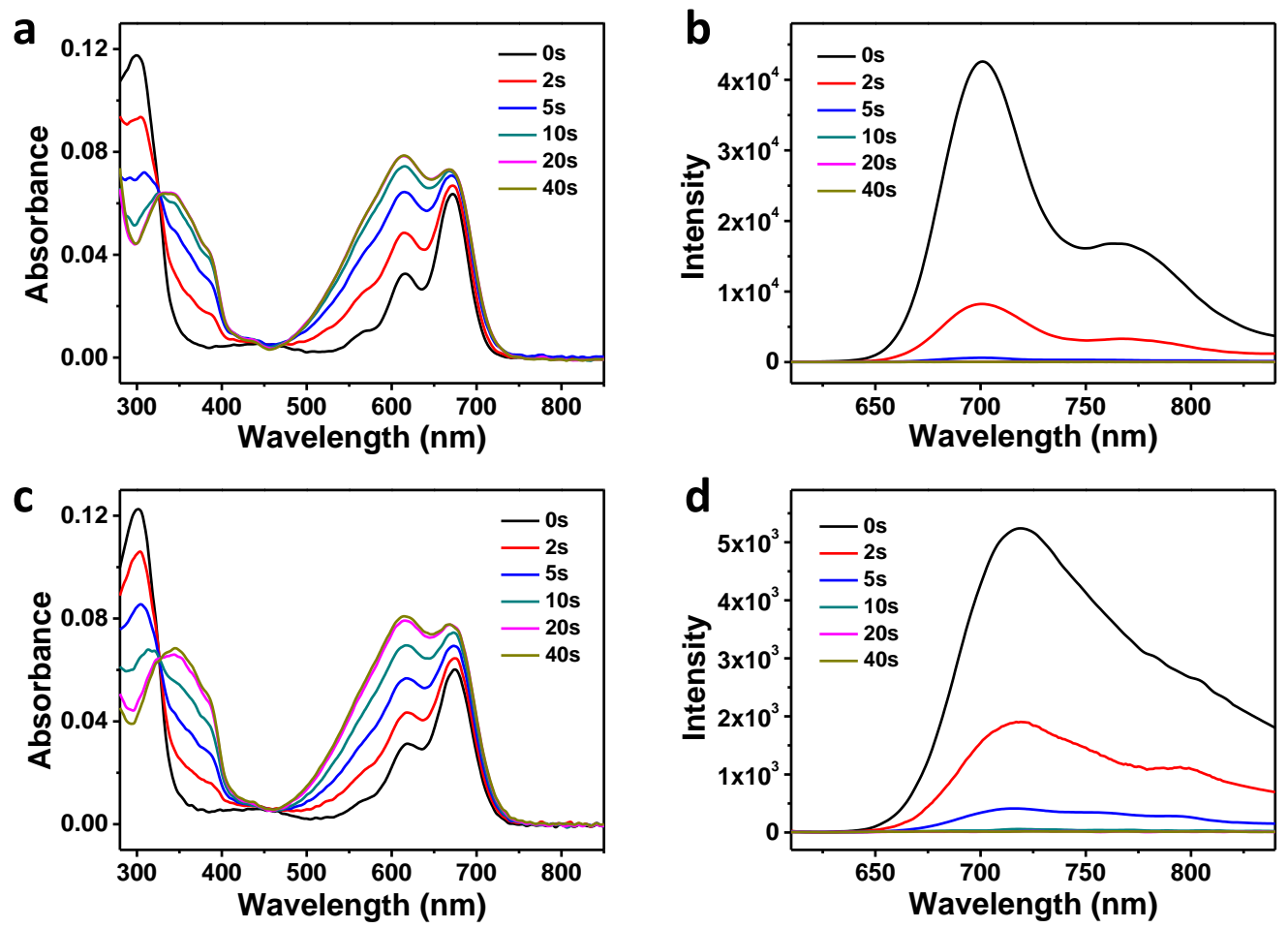

Figure S4. Absorption (a, c) and fluorescence spectra (b, d) changes of TDI-4DTE in toluene and DMF upon $302 \mathrm{~nm}$ ultraviolet irradiation. The concentration is $5 \times 10^{-7} \mathrm{M}$. The excitation wavelength is $600 \mathrm{~nm}$. 

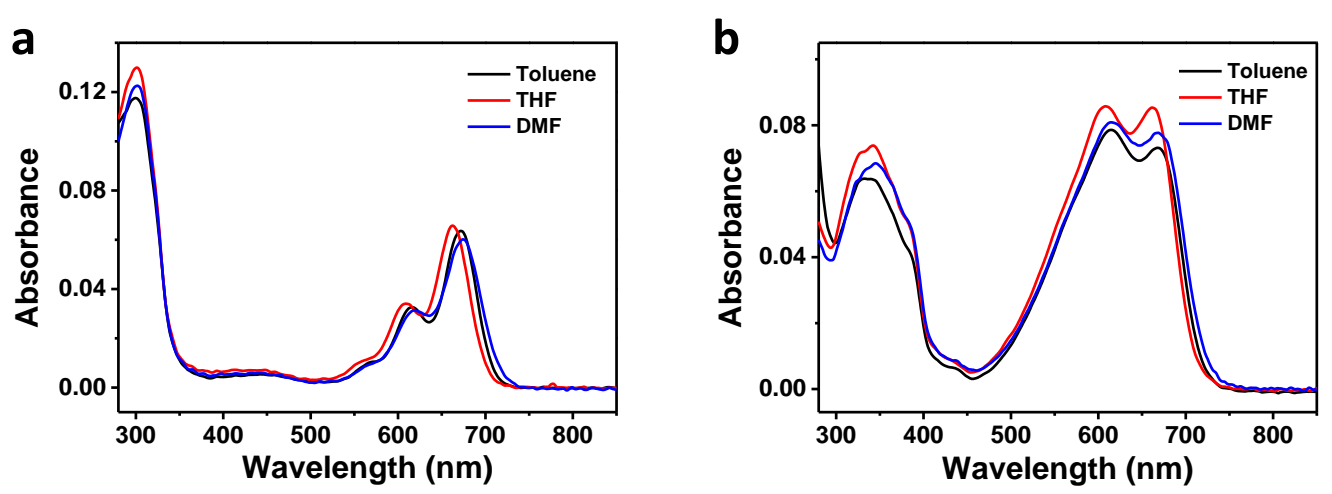

Figure S5. The absorption spectra of (a) the open form and (b) the photostable state of TDI-4DTE in different solvents (Toluene, THF and DMF). The concentrations are $5 \times 10^{-7} \mathrm{M}$.
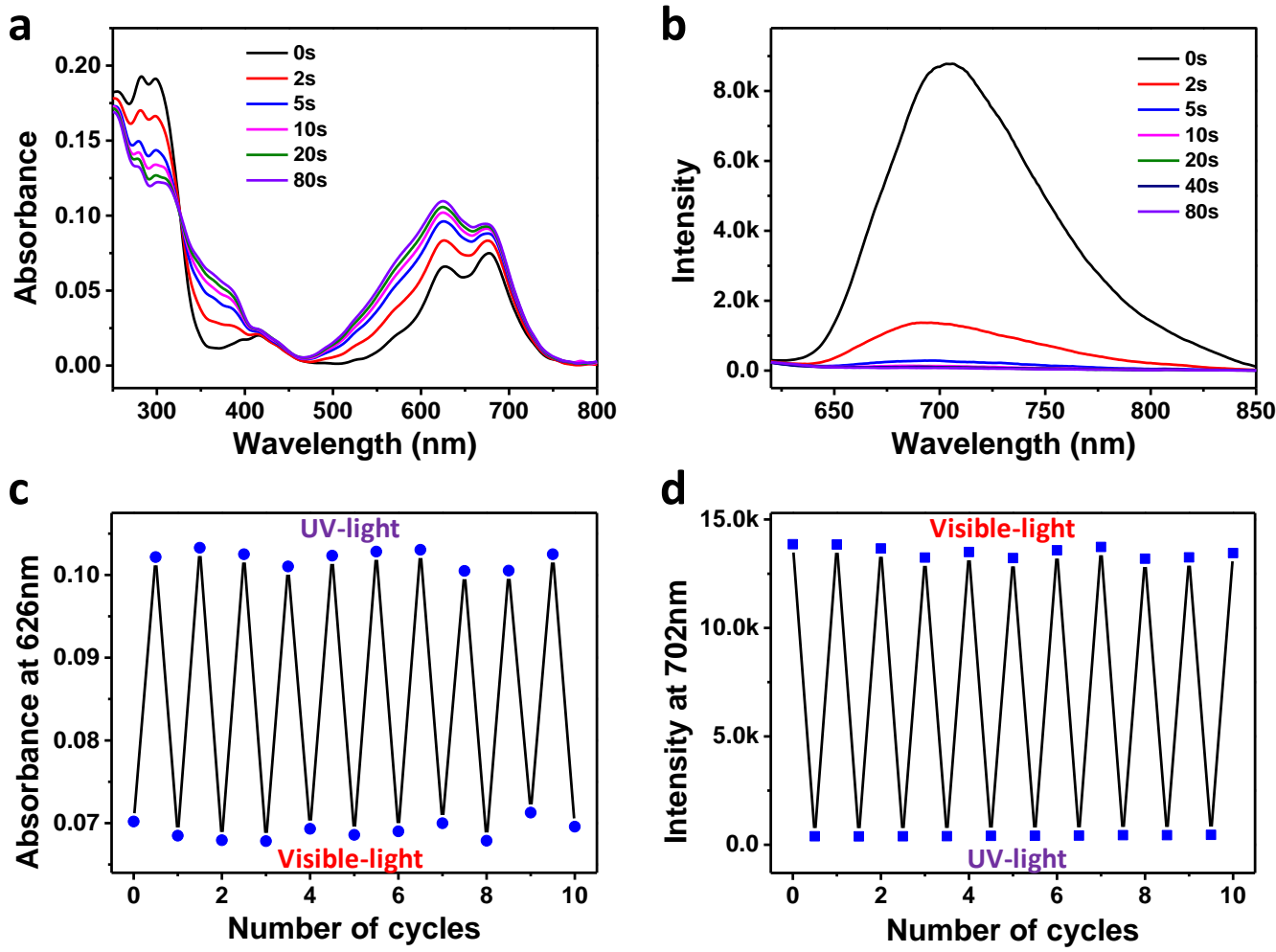

Figure S6. (a) Absorption and (b) fluorescence spectra changes of TDI-4DTE in PMMA film (2 wt \%) upon $302 \mathrm{~nm}$ ultraviolet irradiation; reversible (c) absorbance at $626 \mathrm{~nm}$ and (d) fluorescence intensity at $702 \mathrm{~nm}$ switching for TDI-4DTE in PMMA film (2 wt \%) upon alternating irradiation with UV light (302 nm, $5 \mathrm{~s})$ and visible light $(621 \mathrm{~nm}, 6 \mathrm{~min})$. The excitation wavelength is $600 \mathrm{~nm}$. 

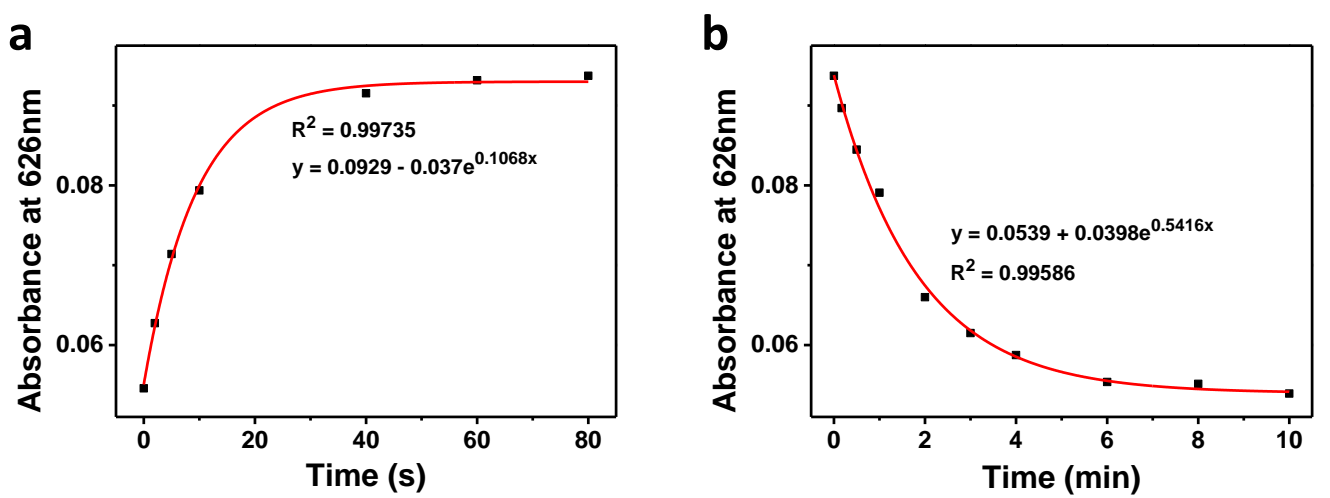

Figure S7. Time-resolved spectroscopic monitoring of (a) the forward and (b) the backward photochromic interconversions between open and closed forms of TDI-4DTE PMA films.
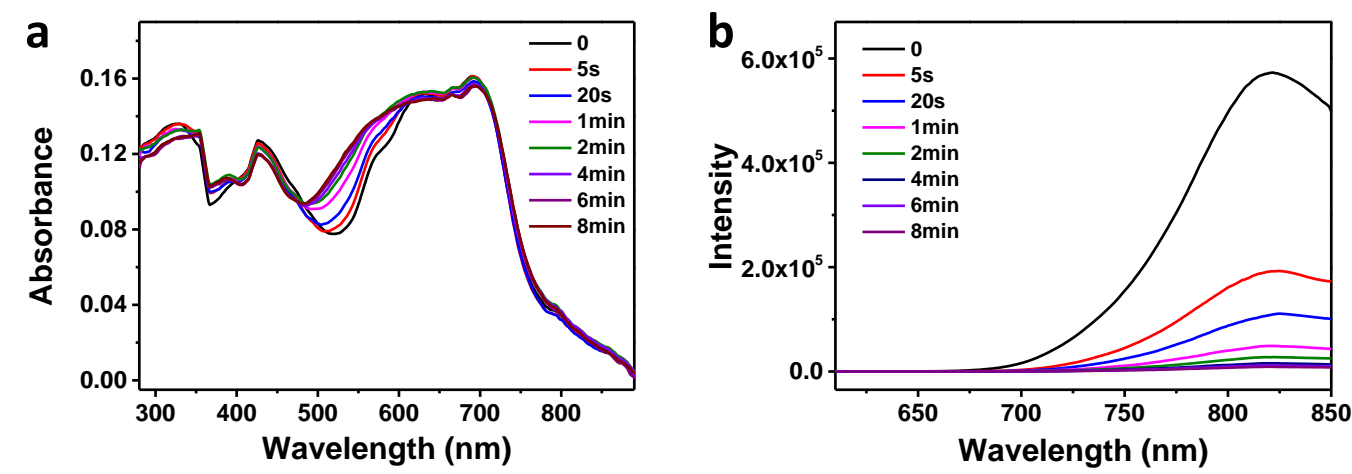

Figure S8. (a) Absorption and (b) fluorescence spectra changes of TDI-4DTE neat solid film upon $302 \mathrm{~nm}$ ultraviolet irradiation. The excitation wavelength is $600 \mathrm{~nm}$. 
Table S1. Optical properties of TDI-4DTE in different medium

\begin{tabular}{|c|c|c|c|c|c|c|}
\hline sample & medium & $\begin{array}{c}\lambda_{\text {abs }} / \mathrm{nm} \\
\text { (Open form) }\end{array}$ & $\begin{array}{c}\lambda_{\text {abs }} / \mathrm{nm} \\
\text { (Closed form) }\end{array}$ & $\lambda_{\mathrm{em}} / \mathrm{nm}$ & $\Phi_{\mathrm{F}}(\%)$ & FL on/off ratio \\
\hline \multirow{6}{*}{ TDI-4DTE } & ${ }^{\text {atToluene }}$ & $301,614,669$ & $340,612,668$ & 702 & 79 & 4810 \\
\hline & ${ }^{\mathrm{a}} \mathrm{THF}$ & $300,609,662$ & $341,608,662$ & 698 & 47 & 3530 \\
\hline & ${ }^{\mathrm{a}} \mathrm{DMF}^{*}$ & $301,617,672$ & $341,616,669$ & 718 & 13.5 & 648 \\
\hline & ${ }^{\mathrm{b}} \mathrm{PMMA}$ & $300,413,626,678$ & 626,676 & 702 & 4.38 & 125 \\
\hline & bPMA & $300,417,626,678$ & $315,626,676$ & 731 & 1.22 & 46 \\
\hline & ${ }^{\mathrm{b}}$ Solid film & $325,427,689$ & 427,695 & 820 & 0.82 & 64 \\
\hline
\end{tabular}

$\lambda_{\text {abs }}$ is the maximum absorption wavelength. $\lambda_{\mathrm{em}}$ is the maximum emission wavelength. Fluorescence on/off ratios is calculated by comparing the intensity at maximum emission wavelength before and after $302 \mathrm{~nm} \mathrm{UV}$ irradiation. $\Phi_{\mathrm{F}}(\%)$ is the fluorescence quantum yields of the open form TDI-4DTE. ${ }^{a}$ The relative fluorescence quantum yields in solutions are estimated using TDI in chloroform $\left(\Phi_{\mathrm{F}}=0.9\right)^{1}$ as standard. ${ }^{\mathrm{b}}$ The absolute fluorescence quantum yields on surrounding films are measured by integrating sphere. * The spectrum from 850 to above $900 \mathrm{~nm}$ is not detected with our limited detection ability of the photomultiplier tube, the fluorescence quantum yield of TDI-4DTE in DMF is estimated by the measured fluorescence integral area.

Table S2. Photocyclization/photocycloreversion quantum yields of TDI-4DTE

\begin{tabular}{|c|c|c|c|c|c|c|c|}
\hline Reaction & Compound & $\lambda_{\text {irr }}$ & $\kappa_{\mathrm{eq}}$ & $\varepsilon_{\mathrm{irr}}\left(\mathrm{M}^{-1} \mathrm{~cm}^{-1}\right)$ & $\alpha_{\mathrm{pss}}$ & $\mathrm{I}\left(\mathrm{mWcm}^{-2}\right)$ & $\Phi_{\mathrm{o} \rightarrow \mathrm{c}} / \Phi_{\mathrm{c} \rightarrow \mathrm{o}}$ \\
\hline Cyclization & TDI-4DTE-O & $302 \mathrm{~nm}$ & 0.41347 & 263000 & 0.9432 & 1.84 & 0.4105 \\
\hline Cycloreversion & TDI-4DTE-C & $621 \mathrm{~nm}$ & 0.00622 & 177500 & 0 & 6.31 & 0.0124 \\
\hline
\end{tabular}

$\lambda_{\text {irr }}$ is the irradiation wavelength. $k_{\text {eq }}$ is the rate constant of equilibration, which is fitted with a monoexponential curve from the kinetics of reequilibration from an arbitrary initial photostationary state $\left(\alpha_{0}\right)$ to a new photostationary state $\left(\alpha_{\mathrm{pss}}\right)$. $\varepsilon_{\text {irr }}$ is the molar extinction coefficient at given wavelengths. $\alpha_{\mathrm{pss}}$ is the fractional population of closed form in PSS irradiated under given wavelengths. $\Phi_{0 \rightarrow d} / \Phi_{c \rightarrow 0}$ is the Cyclization/Cycloreversion quantum yield. 


\subsection{Emission quenching mechanisms of TDI-4DTE}

Fluorescence resonance energy transfer (FRET): To evaluate the efficiency of fluorescence resonance energy transfer from the photoexcited TDI fluorophore to the closed-ring DTE units in TDI-4DTE-C, we calculated the Förster's radius $\left(\mathrm{R}_{0}\right)$ of this compound using equation $\mathrm{S} 1^{2}$.

$$
R_{0}=\left(8.8 \times 10^{-5} \kappa^{2} n^{-4} \Phi_{D} J\right)^{\frac{1}{6}} \quad(\AA) \quad(\text { Equation } \mathrm{S} 1)
$$

The following parameters were used in this calculation: (1) $\Phi_{\mathrm{D}}$ is the fluorescence quantum yield of the TDI-4DTE; (2) $n$ is the refractive index of the medium (parameter: $n^{-4}$, range $\left.\cong 1 / 3-1 / 5\right) ;(3) \kappa^{2}$ is the orientation factor between TDI and DTE transition moments (assumed to be $2 / 3$, valid for rapidly reorienting donor and acceptor); (4) $\mathrm{J}$ is the integral expressing the overlap between the donor emission and acceptor absorption. In this way, we obtained a value of $\mathrm{R}_{0}=10.1 \AA$ for TDI-4DTE-C, and the center-to-center separation distance $\left(r_{d a}\right)$ between TDI and DTE units determined by DFT optimization of the TDI-4DTE-C geometry is $11.9 \AA$. By introducing these parameters into equation $\mathrm{S}^{2}$, the efficiency of the FRET process $(\mathrm{E})$ should be $27.2 \%$.

$$
E=\left[1+\left(\frac{r_{d a}}{R_{0}}\right)^{6}\right]^{-1} \quad(\text { Equation } \mathrm{S} 2)
$$



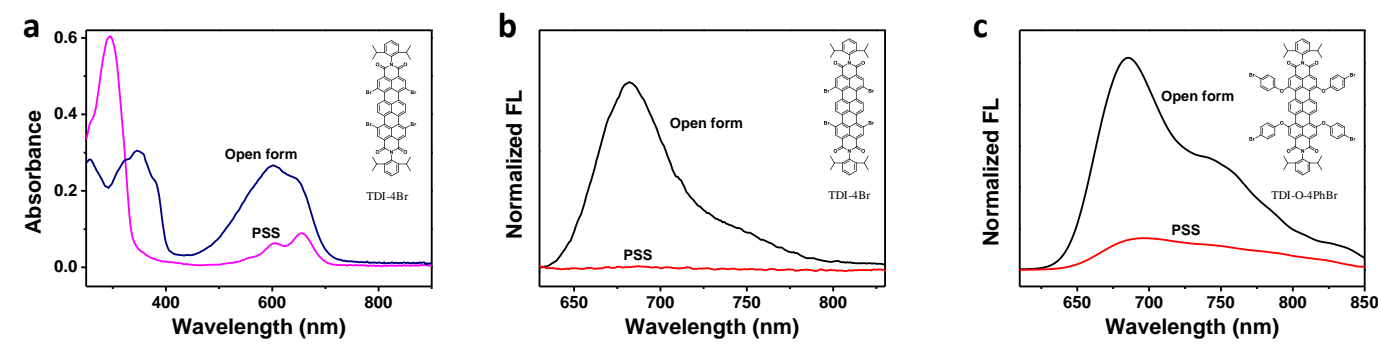

Figure S9. The absorption spectra (a) and fluorescence spectra changes (b) of TDI-4Br and $\mathrm{C}_{8} \mathrm{H}_{17} \mathrm{O}-\mathrm{Ph}-\mathrm{DTE}-\mathrm{Ph}-\mathrm{OH}$ (DTE) which are blended into PMMA film; (c) the fluorescence spectra changes of TDI-O-4PhBr (The compound was prepared according to the reference 1 in the supporting information with slight modification) and $\mathrm{C}_{8} \mathrm{H}_{17} \mathrm{O}-\mathrm{Ph}-\mathrm{DTE}-\mathrm{Ph}-\mathrm{OH}$ (DTE) which are blended into PMMA film upon $302 \mathrm{~nm}$ UV irradiation.
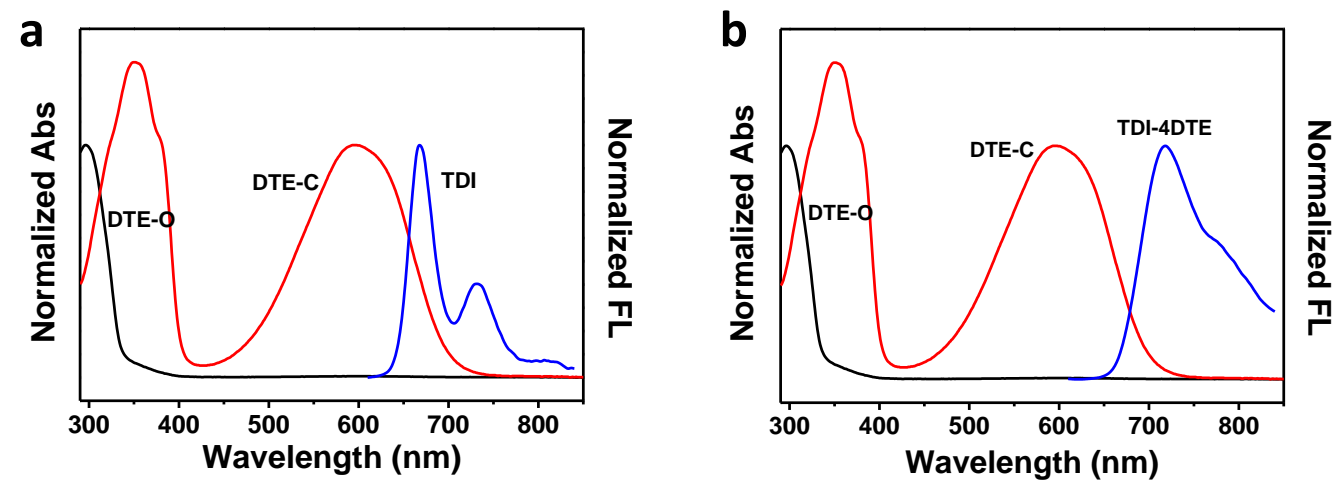

Figure S10. The comparision of (a) absorption of $\mathrm{C}_{8} \mathrm{H}_{17} \mathrm{O}-\mathrm{Ph}-\mathrm{DTE}-\mathrm{Ph}-\mathrm{OH}$ (DTE) and emission spectra TDI and (b) absorption of $\mathrm{C}_{8} \mathrm{H}_{17} \mathrm{O}-\mathrm{Ph}-\mathrm{DTE}-\mathrm{Ph}-\mathrm{OH}$ (DTE) and emission spectra TDI-4DTE. For the model of TDI and DTE, the value of $\mathrm{R}_{0}$ is 14.8 $\AA$ for TDI-4DTE-C and the efficiency of the FRET process (E) measures to be $78.7 \%$. 
Photoinduced electron transfer (PET): To evaluate the efficiency of photoinduced electron transfer in TDI-4DTE, we estimated the change in free energy of this process $\left(\Delta \mathrm{G}^{0}\right)$ using the Weller equation $\mathrm{S} 3^{3}$.

$\Delta G^{0}=e\left[E_{o x}(D)-E_{r e d}(A)\right]-E_{00}-\frac{e^{2}}{4 \pi \varepsilon_{0} \varepsilon_{s} R_{c c}}-\frac{e^{2}}{8 \pi \varepsilon_{0}}\left(\frac{1}{r^{+}}+\frac{1}{r^{-}}\right)\left(\frac{1}{\varepsilon_{r e f}}-\frac{1}{\varepsilon_{s}}\right)$ (eV) (Equation S3) Energy gaps $\left(\Delta \mathrm{G}^{0}\right)$ for the intramolecular charge separation of both isomers of TDI-4DTE in the excited state in a given solvent were estimated with using model $\mathrm{C}_{8} \mathrm{H}_{17} \mathrm{O}-\mathrm{Ph}-\mathrm{DTE}-\mathrm{Ph}-\mathrm{OH}$ and TDI. Since all of our measurements were performed in same solvent, i.e. dichloromethane $\left(\varepsilon_{\mathrm{s}}=\varepsilon_{\text {ref }}\right)$, no estimations for the effective ionic radii of the donor radical cation $\mathrm{r}^{+}$and acceptor radical anion $\mathrm{r}^{-}$are needed, and thus the solvent related fourth term in the Rehm-Weller equation can be neglected for this particular case.

$$
\Delta G^{0}=e\left[E_{\text {ox }}(D)-E_{\text {red }}(A)\right]-E_{00}-\frac{e^{2}}{4 \pi \varepsilon_{0} \varepsilon_{s} R_{c c}} \quad \text { (Equation S4) }
$$

The following parameters were used in this calculation: (1) $\mathrm{E}_{\mathrm{ox}}(\mathrm{D})=1.21 \mathrm{~V}$ and $\mathrm{E}_{\text {red }}(\mathrm{A})=-1.22 \mathrm{~V}\left(\right.$ TDI-4DTE-O); $\mathrm{E}_{\text {ox }}(\mathrm{D})=0.75 \mathrm{~V}$ and $\mathrm{E}_{\text {red }}(\mathrm{A})=-1.16 \mathrm{~V}$ (TDI-4DTE-C); which are according to the first oxidation potentials of the donor DTE and the first reduction potentials of the acceptor TDI unit in TDI-4DTE; (2) $\mathrm{E}_{00}=1.86$ $\mathrm{eV}$ is the excited state energy of the TDI fluorophore; (3) $\mathrm{R}_{\mathrm{cc}}=1.23 \mathrm{~nm}$ (TDI-4DTE-O) and $1.19 \mathrm{~nm}$ (TDI-4DTE-C) are the center-to-center distances between the TDI and DTE groups determined upon DFT geometrical optimization of TDI-4DTE with the B3LYP/6-31G(d) basisset in the Gaussian 09 program; (4) $\varepsilon_{0}=1$ and $\varepsilon_{s}\left(\mathrm{CH}_{2} \mathrm{Cl}_{2}\right)=8.93$, where $\varepsilon$ is the dielectric constant of the solvent. By introducing these parameters into equation S4, the free energy of the PET process $\left(\Delta \mathrm{G}^{0}\right)$ should be $0.32 \mathrm{eV}$ for TDI-4DTE-O and $-0.19 \mathrm{eV}$ for TDI-4DTE-C. 

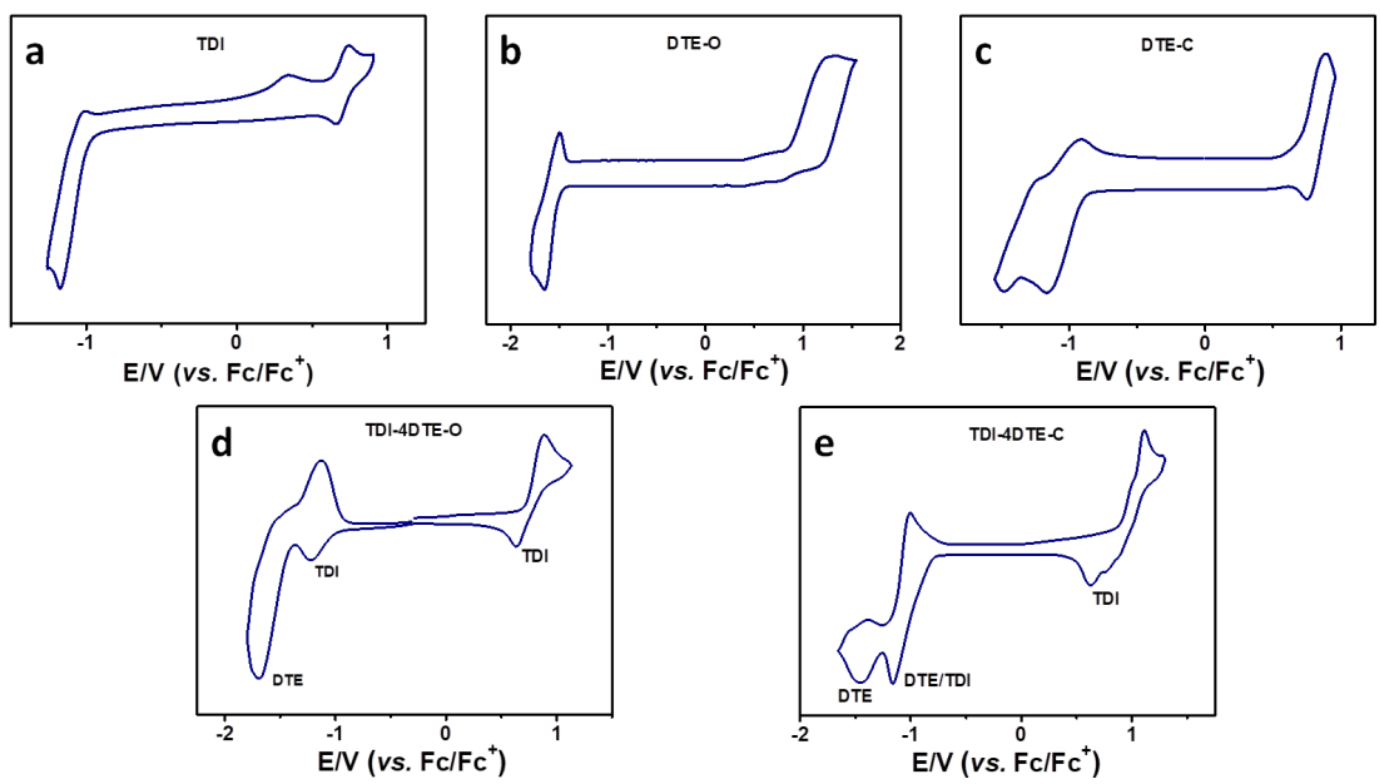

Figure S11. Cyclic voltammograms of (a) TDI, (b) the open form (DTE-O) and (c) the closed form (DTE-C) of $\mathrm{C}_{8} \mathrm{H}_{17} \mathrm{O}-\mathrm{Ph}-\mathrm{DTE}-\mathrm{Ph}-\mathrm{OH}$, (d) TDI-4DTE-O and (e) TDI-4DTE-C in dichloromethane. The supporting electrolyte is TBAHFP $(0.1 \mathrm{M})$, the scan rate is $50 \mathrm{mVs}^{-1}$ and the concentration is $2 \times 10^{-4} \mathrm{M}$.

Table S3. Potentials for the oxidation and reduction processes

\begin{tabular}{|c|c|c|c|c|c|}
\hline Potentials & TDI & DTE-O & DTE-C & TDI-4DTE-O & TDI-4DTE-C \\
\hline $\mathrm{E}_{\text {ox }}(\mathrm{V})$ & 0.66 & 1.21 & 0.75 & 0.63 & 0.63 \\
\hline $\mathrm{E}_{\mathrm{red}}(\mathrm{V})$ & -1.18 & -1.65 & $-1.16,-1.48$ & $-1.22,-1.69$ & $-1.16,-1.46$ \\
\hline
\end{tabular}

All measurements were carried out in dry dichloromethane $\left(10^{-4} \mathrm{M}\right)$ using $\mathrm{Fc} / \mathrm{Fc}^{+}$as an internal standard and TBAHFP $(0.1 \mathrm{M})$ as supporting electrolyte at a scan rate of $50 \mathrm{mVs}^{-1}$. The potentials measured are consistent with the literatures reported. ${ }^{4,5}$ 


\subsection{Non-volatile optical memory}
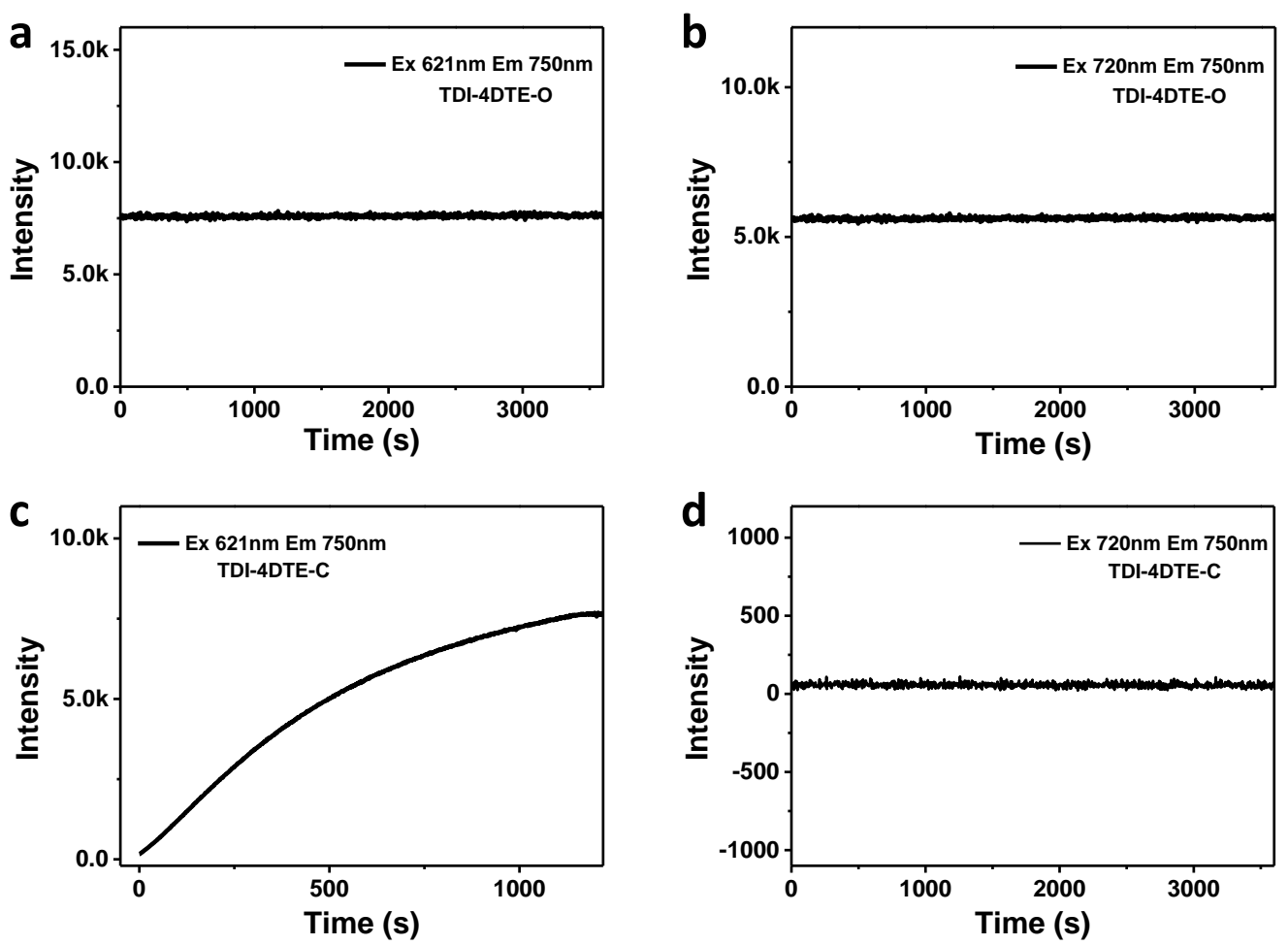

Figure S12. Time-dependent fluorescence intensity at $750 \mathrm{~nm}$ of TDI-4DTE in PMA film upon continuing excitation with monochromatic light at (a, c) $621 \mathrm{~nm}$ and $(\mathrm{b}, \mathrm{d})$ $720 \mathrm{~nm}$ (xenon short arc lamp $450 \mathrm{~W} ; 7.9 \mathrm{mWcm}^{-2}$ for $621 \mathrm{~nm}$ and $5.6 \mathrm{mWcm}^{-2}$ for $720 \mathrm{~nm})$. 

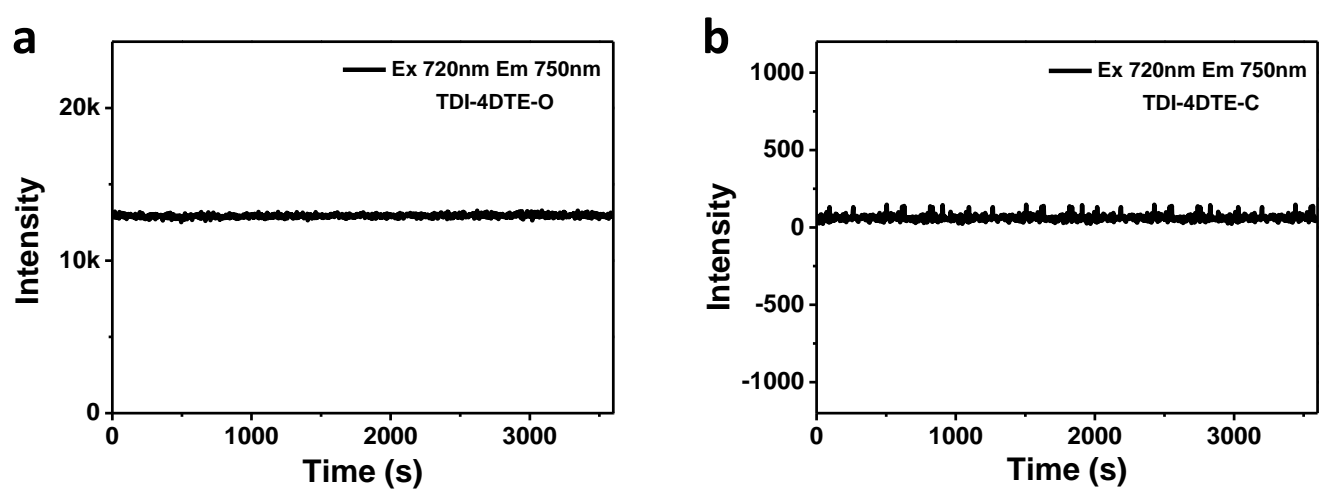

Figure S13. Time-dependent fluorescence intensity at $750 \mathrm{~nm}$ of (a) TDI-4DTE-O and (b) TDI-4DTE-C in PMMA film upon continuing excitation with monochromatic light at $720 \mathrm{~nm}$ (xenon short arc lamp $450 \mathrm{~W} ; 5.6 \mathrm{mWcm}^{-2}$ ).

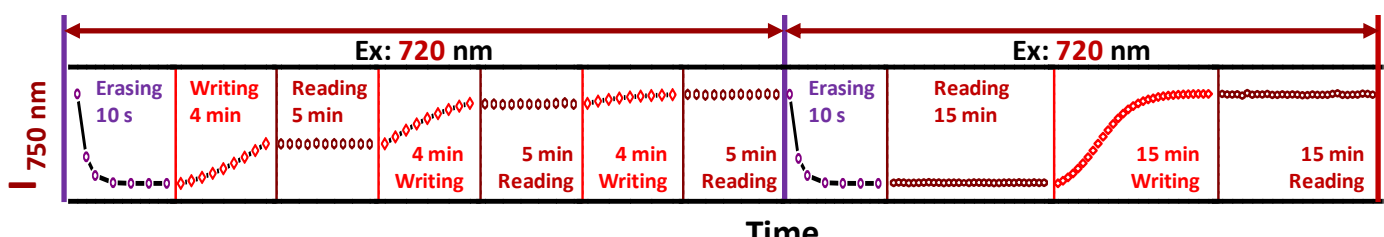

Figure S14. Fluorescence intensity at $750 \mathrm{~nm}$ of erasable fluorescence memory behaviors of the spin-coated TDI-4DTE PMA film (2 wt \%) and its non-destructive readout capability, writing $\left(\lambda_{\mathrm{c} \rightarrow \mathrm{o}}=621 \mathrm{~nm}\right)$, erasing $\left(\lambda_{\mathrm{o} \rightarrow \mathrm{c}}=302 \mathrm{~nm}\right)$ and reading $(\lambda=$ $720 \mathrm{~nm})$. The excitation wavelength is $720 \mathrm{~nm}$. 

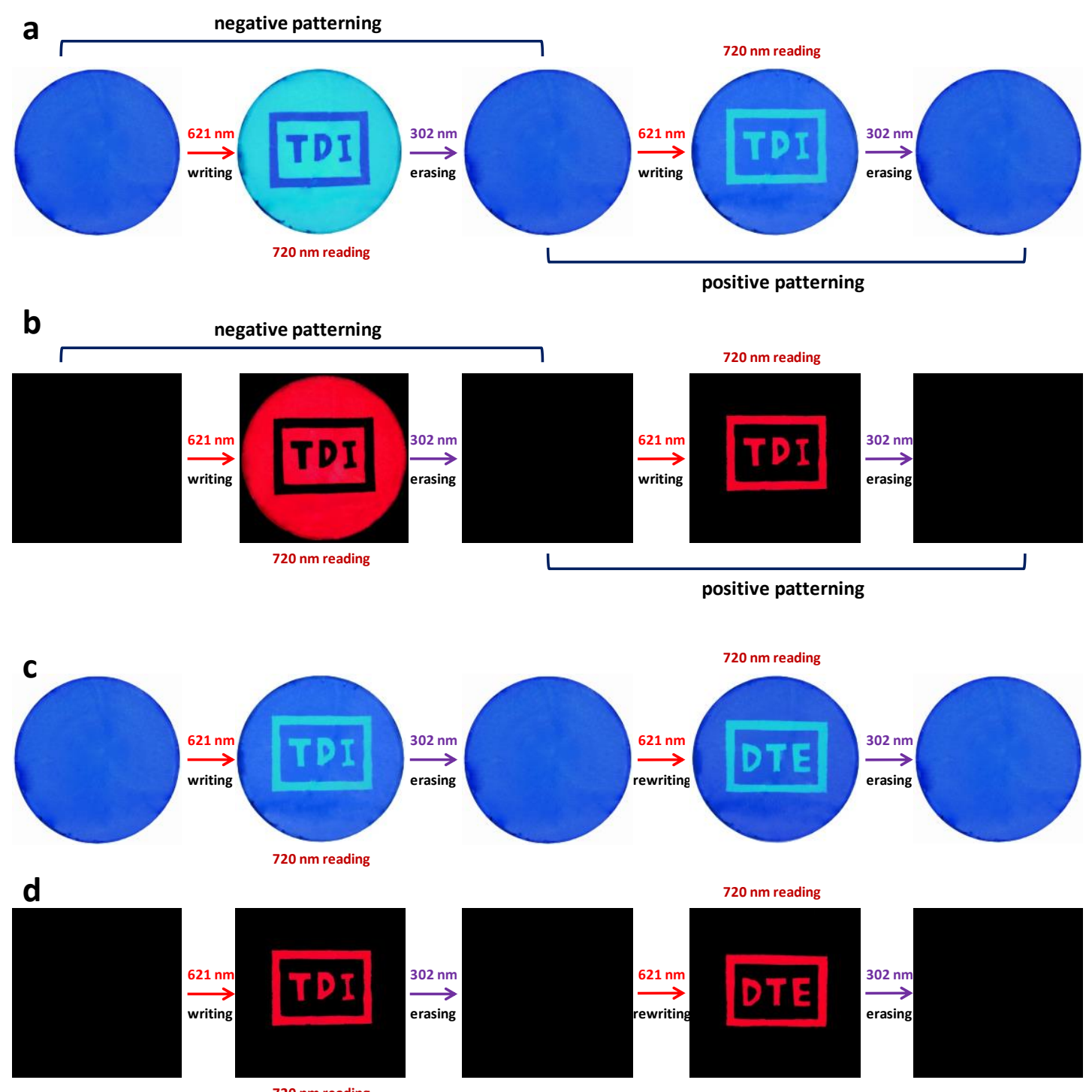

$720 \mathrm{~nm}$ readin
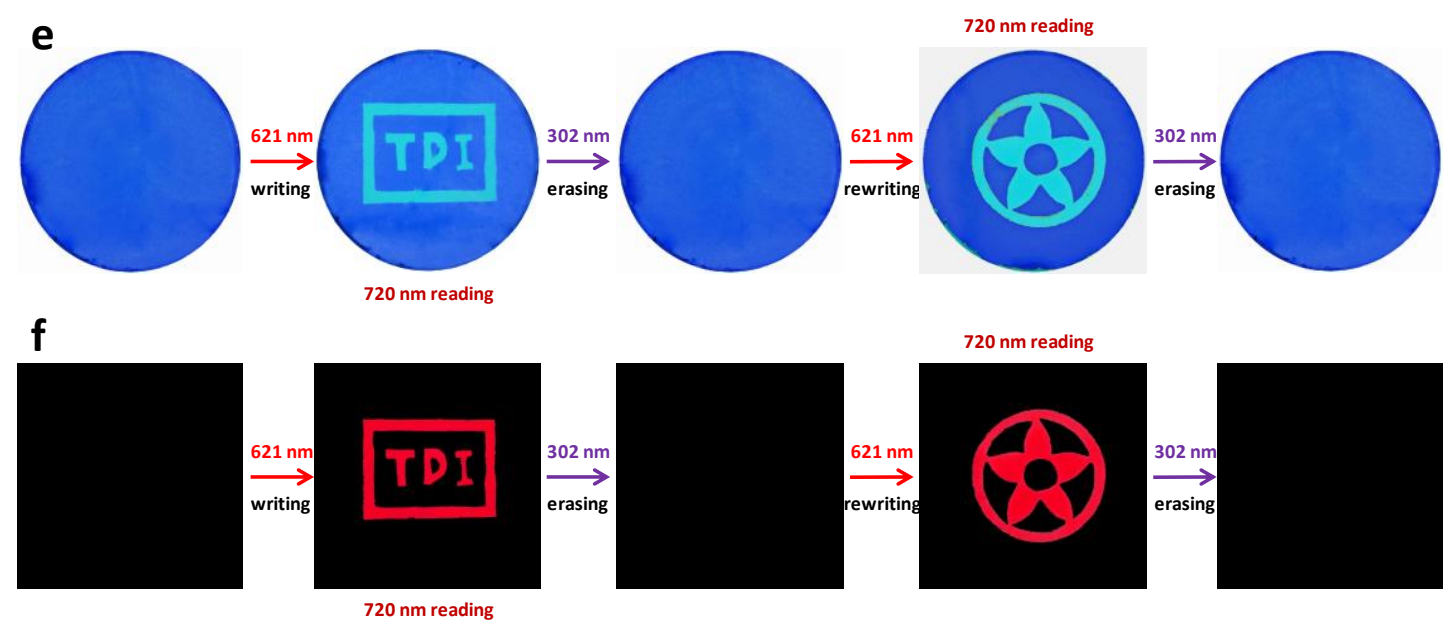

Figure S15. Bright field (a, c, e) and fluorescence (b, d, f) photographs of the spin-coated TDI-4DTE PMA film (2 wt \%) 


\subsection{MS and NMR spectra}

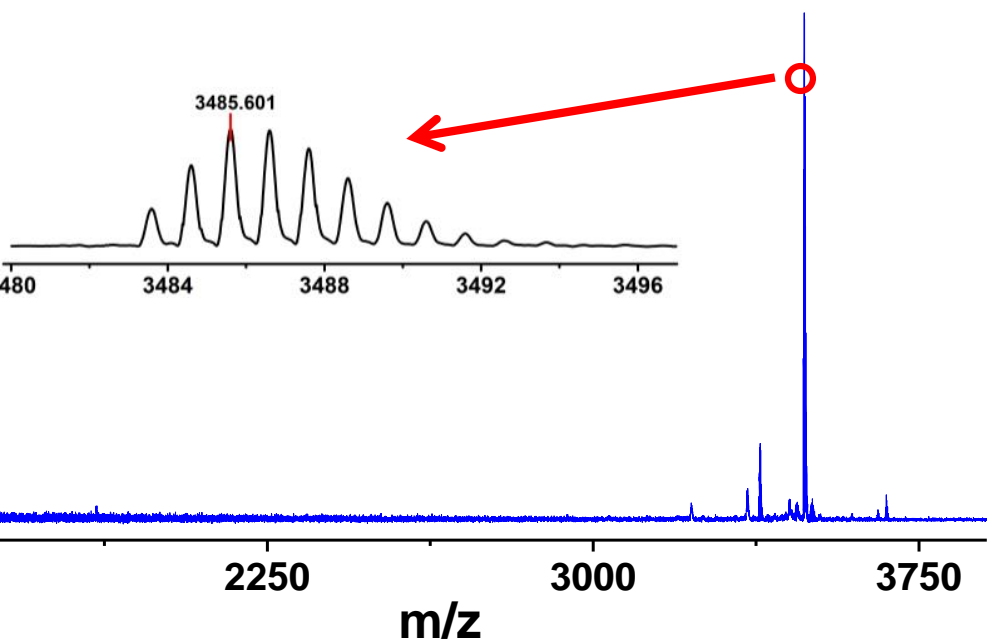

Figure S16. MALDI TOF mass spectra of TDI-4DTE

市

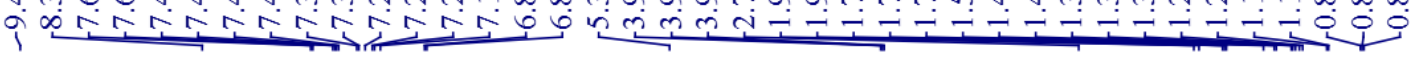
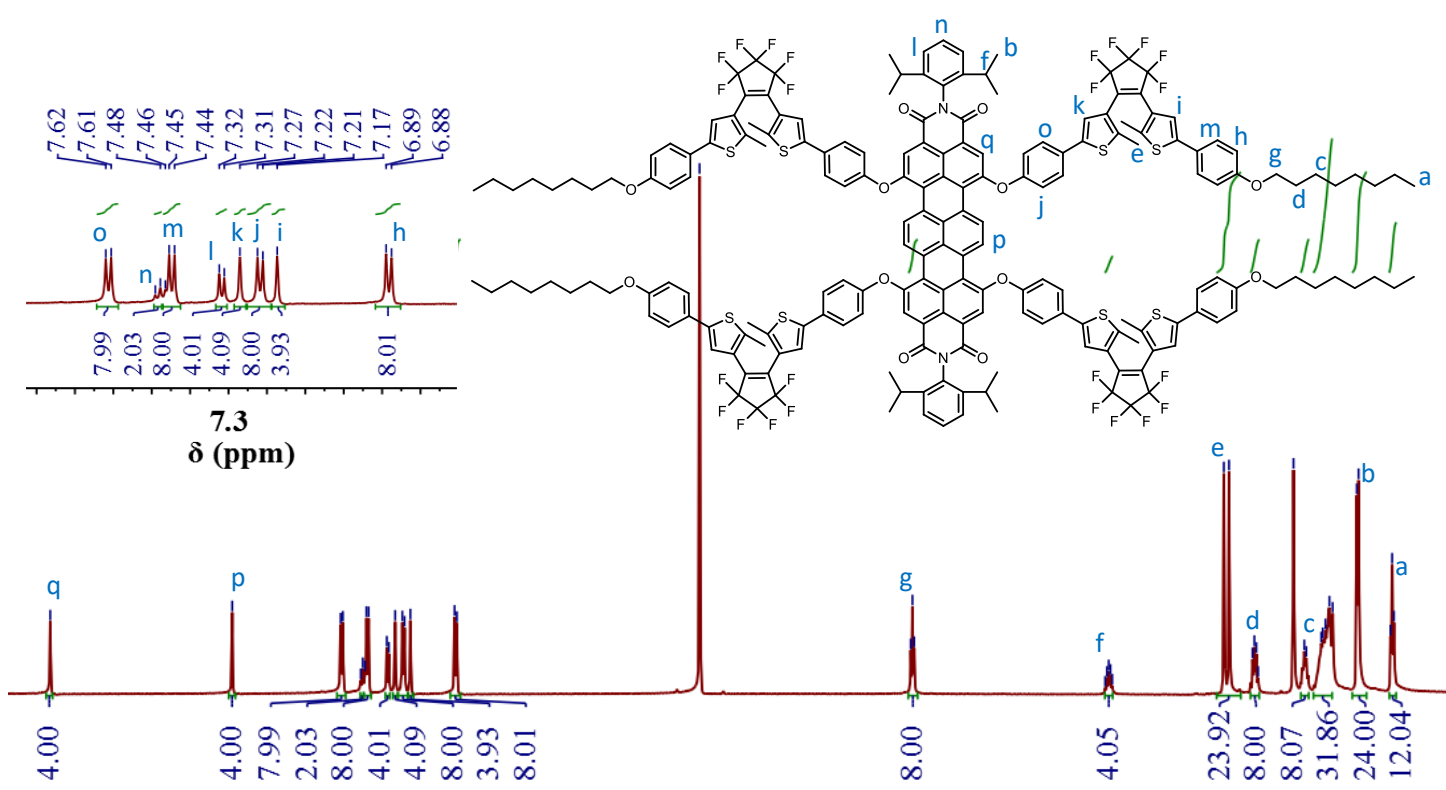

\begin{tabular}{lllllllll}
\hline 9.5 & 8.5 & 7.5 & 6.5 & $\begin{array}{l}5.5 \\
\delta(\mathrm{ppm})\end{array}$ & 4.5 & 3.5 & 2.5 & 1.5
\end{tabular}

Figure S17. ${ }^{1} \mathrm{H}$ NMR spectra of TDI-4DT 


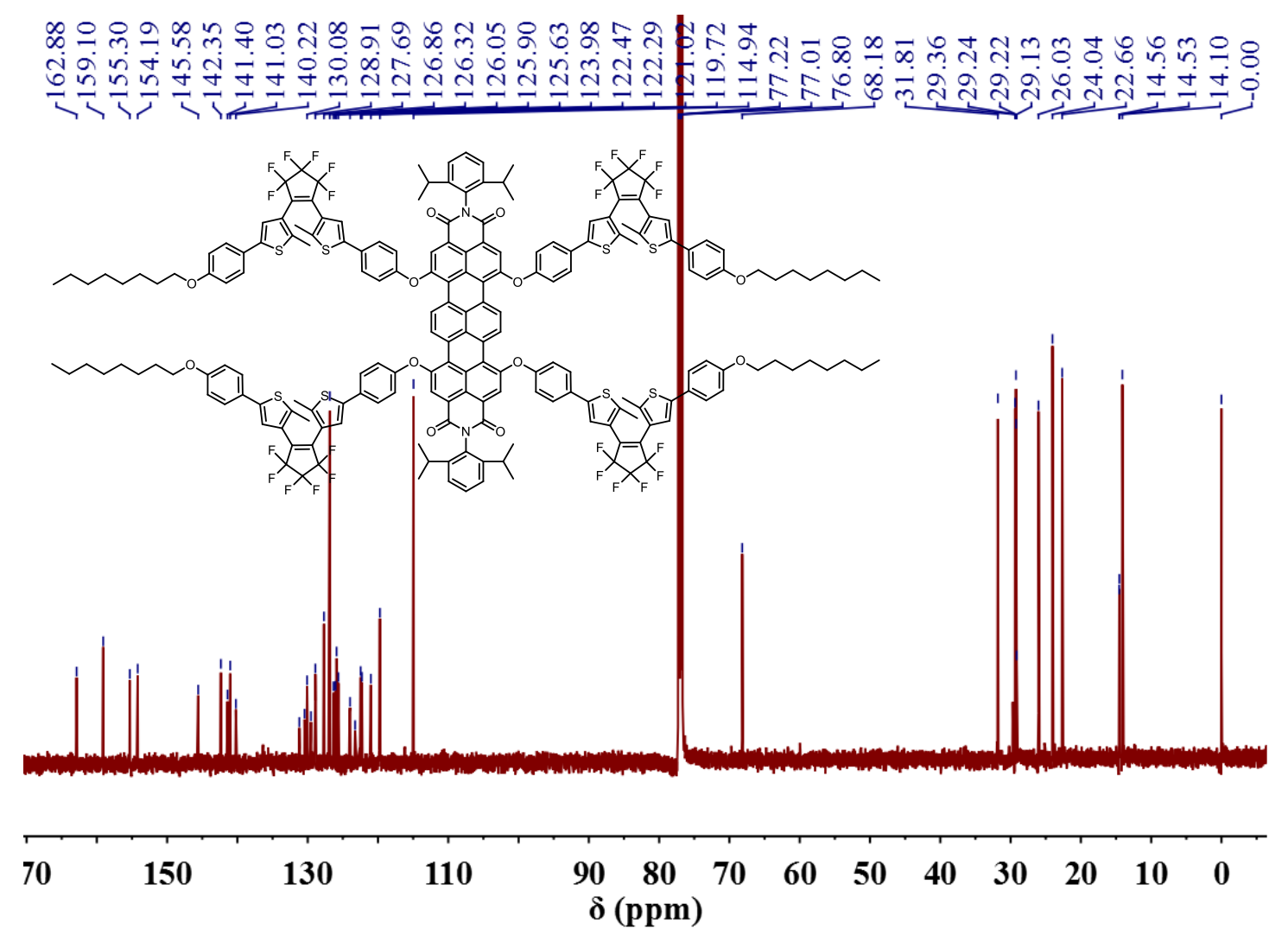

Figure S18. ${ }^{13} \mathrm{C}$ NMR spectra of TDI-4DTE

\section{References}

(1) Xie, N.-H.; Li, C.; Liu, J.-X.; Gong, W.-L.; Tang, B. Z.; Li, G.; Zhu, M.-Q. The Synthesis and Aggregation-Induced Near-Infrared Emission of Terrylenediimide-Tetraphenylethene Dyads. Chem. Commun. 2016, 52, 5808-5811.

(2) Li, C.; Yan, H.; Zhao, L.-X.; Zhang, G.-F.; Hu, Z.; Huang, Z.-L.; Zhu, M.-Q. A Trident Dithienylethene-Perylenemonoimide Dyad with Super Fluorescence Switching Speed and Ratio. Nat. Commun. 2014, 5, 5709.

(3) Xu, K.; Zhao, J.; Cui, X.; Ma, J. Switching of the Triplet-Triplet-Annihilation Upconversion with Photoresponsive Triplet Energy Acceptor: Photocontrollable Singlet/Triplet Energy Transfer and Electron Transfer. J. Phys. Chem. A 2015, 119, $468-481$. 
(4) Berberich, M.; Würthner, F. Terrylene Bisimide-Diarylethene Photochromic Switch. Chem. Sci. 2012, 3, 2771-2777.

(5) Browne, W. R.; de Jong, J. J. D.; Kudernac, T.; Walko, M.; Lucas, L. N.; Uchida, K.; van Esch, J. H.; Feringa, B. L. Oxidative Electrochemical Switching in Dithienylcyclopentenes, Part 1: Effect of Electronic Perturbation on the Efficiency and Direction of Molecular Switching. Chem. - Eur. J. 2005, 11, 6414-6429. 